\title{
The effectiveness of glass beads for plating cell cultures
}

Alidivinas Prusokas ${ }^{1,2}$, Michelle Hawkins ${ }^{2}$, Conrad A. Nieduszynski ${ }^{3}$ and Renata Retkute $^{4^{*}}$

${ }^{1}$ Plant and Microbial Sciences, School of Natural and Environmental Sciences, University of Newcastle, UK

${ }^{2}$ Department of Biology, University of York, UK

${ }^{3}$ Sir William Dunn school of pathology, University of Oxford, UK

${ }^{4}$ The Zeeman Institute for Systems Biology and Infectious Disease Epidemiology Research (SBIDER), The University of Warwick, UK

* Corresponding author: Renata Retkute, r.retkute@warwick.ac.uk

\section{Abstract}

Cell plating, the spreading out of a liquid suspension of cells on a surface followed by colony growth, is a common laboratory procedure in microbiology. Despite this, the exact impact of its parameters on colony growth has not been extensively studied. A common protocol involves the shaking of glass beads within a petri dish containing solid growth media. We investigated the effects of multiple parameters in this protocol - the number of beads, the shape of movement, and the number of movements. Standard suspensions of Escherichia coli were spread while varying these parameters to assess their impact on colony growth. Results were compared by a number of metrics - the number of colonies, the mean distance between closest colonies, and the variability and uniformity of their spatial distribution. Finally, we devised a mathematical model of shifting billiard to explain the heterogeneities in the observed spatial patterns. Exploring the parameters that affect the most fundamental techniques in microbiology allows us to better understand their function, giving us the ability to precisely control their outputs for our exact needs.

Keywords: Colony Forming Units; cell growth; cell plating; E.coli, shifting billiard 


\section{Introduction}

A prominent technique in microbiology is cell plating to enable colony growth on nutrient agar. The goal of cell plating is to separate cells contained within a small sample volume by homogeneously distributing the cell suspension over the surface of a plate. This results in the formation of discrete colonies after incubation, that can be enumerated or subjected to further analysis. Counting the number of colonies is used in many cell culture protocols: detection of bacteria in food samples or clinical specimens (Goetz, 2006); measuring progenitor stem cell content (Wognum, 2013); constructing gene-knockout libraries (Horie, 2011); cell-based DNA cloning (Strachan, 2011); testing resistance to drugs (Gianyecchio et al, 2019); and studying the evolution of antibiotic resistance (Ragheb et al., 2019). Achieving successful cultivation of cells depends critically on the choice of appropriate growth media, cell plating method, and incubation conditions.

A colony is defined as a visible cluster of cells growing on the surface of a medium, presumably derived from a single cell. These single progenitor cells are also called Colony Forming Units (CFU), which provide an estimate of the number of viable cells. The more homogeneously cells are spread on the surface during plating, the better separated the resulting cell colonies, leading to a more precise CFU estimation. It is expected that the number of colonies formed is linearly proportional to the concentration of viable cells in the suspension, provided that the suspension was mixed and spread well. If the suspension is not spread well, clusters of cells will be formed which visually resemble a single colony and therefore will be enumerated as a single colony (Jennison, 1937); furthermore bias may be introduced when analysing genetic variations and heredity of such clusters of cells (Čepl et al 2016). To proceed with further procedures, the cells need to be restreaked to get single colonies which takes extra incubation time.

There are two strategies for cell plating: spread-plating with a turntable rod or spreadplating with glass beads (Hartman, 2011; Sanders, 2012). Using the first method, a small volume of a cell suspension is spread over the plate surface using a sterile bent glass rod as the spreading device. The second method, which is the subject of our study, involves shaking glass beads over the surface of the plate. This technique is also known as the Copacabana method (Worthington, 2001; Mills, 2005).

The protocol for using sterile glass beads for dispersion of cells on solid media has the following steps (Sanders, 2012; Worthington, 2001): (1) a cell suspension is dispensed 
onto the middle of a round Petri dish containing solid media; (2) spherical glass beads are poured into the middle of the plate; (3) the plate lid is closed; (4) the plate is agitated with a shaking motion so that the glass beads roll over the entire surface of the plate; $(5)$ the plate is then inverted to remove the beads before incubation.

In this study we investigate how the way a plate is agitated influences the spread of colonies. We perform experiments with the bacteria E.coli and use a model from statistical mechanics - billiard, to explain heterogeneities in the observed spatial patterns. Billiard is a type of mathematical model describing a dynamical system where one or more particles moves in a container and collides with its walls (Chernov, 2006). We introduce a new class of billiard - shifting billiard, which serves as a conceptual abstraction of the cell plating with glass beads.

\section{Materials and Method}

\section{Cell plating experiment}

The experiments were performed on circular plates with $88 \mathrm{~mm}$ diameter. Sterile $4 \mathrm{~mm}$ glass plating beads (manufactured by Sigma-Aldrich) were used for the dispersion of cells over the surface of a plate. This gives the ratio between glass bead and plate $d=4 / 88=0.045$.

We explored following movement of a plate:

- L-shape. Plate is moved on a trajectory resembling the horizontally reflected letter "L": up- right-left-down.

- Up-down. Plate is moved up and down along the vertical axes.

- Hourglass. Plate is moved on a trajectory resembling the hourglass: diagonal up-left-diagonal down-right.

We consider a single loop as a set of plates moves that gets the plate into the same initial position.

E. coli K-12 MG1655 was incubated in LB broth overnight. LB broth had been set to $\mathrm{pH} 7.05$ via the addition of $\mathrm{NaOH}, 5 \mathrm{ml}$ of which was aliquoted to a $50 \mathrm{ml}$ CellStar tube. A single $E$. coli colony was sampled from a streaked plate, placed within the LB broth, and incubated in a $37^{\circ} \mathrm{C}$ shaker overnight until saturation. This overnight stock was serially diluted by factors of 10 in $56 / 2$ salts to the required dilution. $100 \mu$ l of the cell dilution was plated on LB agar $1.8 \% \mathrm{~W} / \mathrm{V}$, utilising the above motions, with a varying 
number of loops and beads. These plates were incubated overnight at $37^{\circ} \mathrm{C}$, and subsequently imaged.

\section{Image analysis}

Colony counts were obtained automatically from imaging data, utilising custom code. The workflow of image analysis is shown in Figure 1. First, each image is binarized with a low threshold in order to find the boundaries of a plate; this gives the scaling factor for transforming pixels into millimeters. Next, we extract single-channel images corresponding to each of the color channels in the original image. We have found that working with the image in the red channel improved detection of colonies. Finally, we fit centroids representing colonies by varying the threshold of binarization. The code is provided as Supplementary Material.

\section{Assessing the effectiveness of plating method}

We used the following metrics to assess plating methods: the number of colonies, $n_{C F U}$; the normalised mean distance to nearest colony, $d_{N B}$; and the variability and uniformity in colony spatial distribution on a plate, $C V_{S}$ and $m_{S}$ respectively. To normalise the mean distance to nearest colony, we looked for the radius of colony, $r$, such that identical colonies with radius $r$ could fill the plate uniformly. The cumulative area of such colonies must be equal or less than the area of plate, $A$, i.e, $A \leq$ $n_{C F U}\left(\pi r^{2}\right)$. Within these conditions, this radius would be equal to $r=44 / \sqrt{n_{C F U}}$. The normalised mean distance to nearest colony, $d_{N B}$, was then calculated by dividing the observed mean distance to nearest colony by the expected distance between optimally packed colonies, which is $2 * r$. We calculated median as well, but it had a similar values as mean. To determine the spatial variability, we grouped colonies into $5^{\circ}$ intervals, calculated the number of colonies in that arc, and estimated the coefficient of variation between these numbers, $C V_{S}$. Next we calculated the distances of colonies to the center of the plate, binned into a $1 \mathrm{~mm}$ intervals and calculated an average difference between observed frequencies, and expected frequencies, $m_{S}$. Expected frequencies for the latter metric were assumed to follow the triangular distribution. Larger values of $n_{C F U}$ and $d_{N B}$ correspond to a better spread, while smaller values of $C V_{S}$ and $m_{S}$ indicate more uniformly spread of colonies. Figure 2 shows a graphical representation of the metrics, which gives the following values: $n_{C F U s}=335, d_{N B}=$ $0.6, C V_{S}=0.46$ and $m_{S}=2.3$. 


\section{Mathematical model}

Billiard describes a dynamic system where one or more particles move in a container, termed a billiard, and collide with its walls (Chernov, 2006). Billiard systems based on the wave dynamics in cavities, acoustic resonance in water, atoms bouncing off a beam of light and quantum dots have been studied over several decades both experimentally and theoretically (Nagler et al., 2007).

We set the radius of the circular billiard to one. Let $\Gamma$ denote the unit disk, and $\partial \Gamma$ denote its boundary. Particle movement within a billiard is defined by the elastic collision rule: the angle of reflection is equal to the angle of incidence (Berry, 1981; Chernov \& Markarian, 2006). Denote by $q_{t}=\left(x_{t}, y_{t}\right)$ the coordinates of the moving particle at time $t$, and by $v_{t}=\left(u_{t}, w_{t}\right)$ its velocity vector. Then its position at time $t+$ $\Delta t$ can be computed by

$$
\begin{aligned}
& x_{t+\Delta t}=x_{t}+\Delta t u_{t}, \\
& y_{t+\Delta t}=y_{t}+\Delta t w_{t} .
\end{aligned}
$$

When the particle collides with the boundary $\partial \Gamma$, its velocity vector $v$ gets reflected across the tangent line to $\partial \Gamma$ at the point of collision and the new post-collision velocity vector can be computed as

$$
v^{\text {new }}=v^{\text {old }}-2<v^{\text {old }}, n>n,
$$

where $n$ is the unit normal vector to the boundary and $\langle v, n\rangle$ denotes the scalar product.

For an arbitrary collision between two particles, the post-collisional velocities are given by:

$$
\begin{aligned}
& v_{1}^{\text {new }}=v_{1}^{\text {old }}+0.5(1+r)\left(v_{2}^{\text {old }}-v_{1}^{\text {old }}\right) \cdot k k, \\
& v_{2}^{\text {new }}=v_{2}^{\text {old }}+0.5(1+r)\left(v_{2}^{\text {old }}-v_{1}^{\text {old }}\right) \cdot k k,
\end{aligned}
$$

where $0 \leq r<1$ is the normal coefficient of restitution, which is typically 0.8 for glass beads (Lorenz et al., 1997); v. $k$ denotes the vector product, and $k$ is the collision vector, directed from the centre of the second particle to that of the first particle:

$$
k=\left(q_{1}-q_{2}\right) /\left\|q_{1}-q_{2}\right\| .
$$

We have the following set-up for our model: there are $n$ glass beads, which at a time $t$ are randomly distributed within a unit circle in such a way that they do not overlap, i.e the Euclidean distance between beads centers is larger than $2 d$. We assume that 
the plating dish is moved a unit distance (i.e. corresponding to the diameter of a plate). All particles are stationary at the start of the simulation, and start moving after collision with the moving boundary $\partial \Gamma$. The code is provided as Supplementary Material.

\section{Results}

First, cell plating was carried out with varying concentrations of E.coli cells. We used the "L-shape" movement with 10 glass beads, and either 5 or 10 loops. Results from this experiment are shown in Table 1. As cell concentration decreased, the normalised mean distance between closest colonies, $d_{N B}$, and spatial variability of colonies, $C V_{S}$, increased. The number of colonies was $950-1050$ for $10^{-5}$ dilution, $280-380$ for $10^{-6}$ dilution, and $50-70$ for $10^{-7}$ dilution. It is suggested that for manual counting, the best range of a number of colonies should be between 30 and 300 (Tomasiewicz et al., 1980). Therefore, for further analysis we have chosen the $10^{-6}$ dilution as this is close to the suggested range, while reducing the stochasticity a smaller number of colonies would introduce.

Table 2 displays a summary from the experiments: each row corresponds to a different method of cell plating, number of glass beads and number of loops. We have analysed 40 configurations in total. We observed that the number of CFUs increased when the number of loops increased from 5 to 25. Most of the experiments produced a consistent number of colonies. The exceptions were for cells spread using either 100 loops, or 25 beads and 25 loops, which gave much lower colony numbers. Methods producing the smallest number of colonies also had the largest values of $d_{N B}$ and $C V_{S}$.

We could identify three groups of configurations which produced $n_{C F U}>300$ : (i) 'Lshape' movement with 2-10 beads and 25 loops; (ii) 'L-shape' movement with 10-25 beads and 5 loops; and (iii) 'up-down' movement with 3-25 beads and 50 loops. However, the latter configuration performed quite poorly with respect to other metrics, especially $d_{N B}$ and $m_{S}$. In terms of the number of steps the plate is moved, 50 'updown' loops are equivalent to 25 'L-shape' loops. Mean distance to the nearest colony for the 'up-down' motion had the smallest values overall. The 'hourglass' movement performed well for 10 loops and 3-25 glass beads in terms of values of $n_{C F U}$, and had one of the lowest values of spatial variability, $C V_{S}$, however the number of colonies were not as high as for 'L-shape' or 'up-down' trajectories. 
Next, we used the model of shifting billiard to investigate the observed spatial patterns in the 'up-down' movement. Figure 3 shows experimental images of E.coli colonies and density histograms of simulated particle trajectories for the 'up-down' movement. For model simulations with $n$ beads, we have added an extra bead $n$, but kept initial positions of $1 . .(n-1)$ beads the same as in simulations with $n-1$ beads. It can be seen that even with 50 loops, the trajectories of beads follow the movement of the plate. This mimics exactly how colonies are positioned in the experiments, i.e. along and parallel to a vertical axis. As a number of beads is increased, there is a possibility of a glass bead escaping the expected trajectory due to a random collision with other glass beads.

Furthermore, we used this model to explain heterogeneities in the observed spatial patterns when using 'L-shape' movement. Figure 4 shows representative images of E.coli colonies and Figure 5 shows corresponding density histograms of simulated particle trajectories for the 'L-shape' movement. It can be seen from the simulations, that when the number of glass beads or the number of loops is low, the surface of the plate can not be fully explored by the moving beads, and particular areas (top left quarter) is hardly visited by any of glass beads at all. In order to improve surface coverage, it is necessary to increase either the number of beads or the number of loops. For example, 5 glass beads with 25 loops, or 10 glass beads and 10 loops, produced a reasonable coverage of plate surface.

Our model was not able to explain the decrease in the number of colonies when using a very high number of loops. Similar phenomena have been observed when plating central memory cells, which was attributed to cells getting stuck on beads and hence removed during the debeading process (Aksoy et al 2019).

For 'L-shape' movement and 100 loops, the number of CFU decreased gradually from 160 to 20 when the number of glass beads was increased. So this phenomenon was due to a combination of both longer time of plate agitation, and more frequent collisions between glass beads.

We additionally tested this method on plating the yeast Saccharomyces cerevisiae and found that the plate moved using 'L-shape' trajectory with 10 beads and 5-10 loops produced appropriate colony separation (Supplementary materials and Supplementary Figure 1). 


\section{Conclusions}

In this work we characterize the effectiveness of glass beads for plating cell cultures. We examined the number of colonies, mean distance between closest colonies, and variability and uniformity of spatial distribution of colonies. Our results indicate that the Copacabana method is highly efficient, although care needs to be taken when choosing the trajectory of plate movement, number of beads and number of loops.

An exploratory look at the experimental data revealed some interesting attributes that required further investigation using mathematical modelling approach. We introduced shifting billiard as a conceptual abstraction of the cell plating with glass beads. Numerical analysis of the dynamics of this simple yet efficient billiard, indicated a close relationship between plate movement and quality of colony distribution. Simulations show that when the number of glass beads or a number of loops is low, the surface of the plate can not be fully explored by the moving beads. This could be improved by increasing either the number of beads or the number of loops. However, a very high number of loops (i.e. 100) should be avoided, as experimental data showed that the number of colonies was much lower compared to other configurations.

We have analysed the properties of cell spread using digitized images and mathematical modelling. Optical density (OD) readings have been used to evaluate the number of CFU based on the time it takes to reach the predetermined OD of 0.1 when compared with a reference standard curve (Ben Yehezkel et al. 2011). However, this technique would not provide the necessary details for the spatial analysis we performed.

In conclusion, we recommend using 'L-shape' trajectory with 2-10 beads and 25 loops which should produce good colony separation in terms of the number and spatial spread of colonies. Recently, automated systems have been utilised for highthroughput cell handling and analysis (Whitehead et al., 2018). Manual transformation and spreading on agar plates are still in otherwise fully automated liquid handling platforms (Hans et al., 2018). Therefore, we believe that our results should be of interest when using automated platforms and could guide programming of automatic cell plating protocols. 


\section{References}

Aksoy B.A., Czech E., Paulos C., Hammerbacher J. (2019) Computational and experimental optimization of $\mathrm{T}$ cell activation. bioRxiv http://dx.doi.org/10.1101/629857

Ben Yehezkel T., Nagar S., Mackrants D., Marx Z., Linshiz G., Shabi U., Shapiro E. (2011) Computer-aided high-throughput cloning of bacteria in liquid medium. Biotechniques 50(2): 124-7.

Berry MV. (1981) Regularity and chaos in classical mechanics, illustrated by three deformations of a circular billiard. Euopean Journal of Physics 2, 91-102.

Čepl J., Blahůšková A., Neubauer Z., Markoš A. (2016) Variations and heredity in bacterial colonies. Commun Integr Biol. 9 e1261228.

Chernov NI, Markarian R. (2006) Chaotic Billiards. American Mathematical Society.

Gianyecchio C, Lozano NA, Henderson C, Kalhori P, Bullivant A, Valencia A, Su L, Bello G, Wong M, Cook E, Fuller L, Neal JB, Yeh PJ. (2019) Variation in Mutant Prevention Concentrations. Frontiers in microbiology 10.

Goetz F, Bannerman T, Schleifer KH. (2006) The Genera Staphylococcus and Macrococcus. In: A Handbook on the Biology of Bacteria, Vol 4, Third Edition: Bacteria: Firmicutes, Cyanobacteria. Springer; 5-75.

Hans S., Gimpel M., Glauche F., Neubauer P., Cruz-Bournazou M.N..Automated Cell Treatment for Competence and Transformation of Escherichia coli in a HighThroughput Quasi-Turbidostat Using Microtiter Plates. Microorganisms. 25; 6(3) E60. Hartman D. (2011) Perfecting Your Spread Plate Technique. J Microbiol Biol Educ. 12(2): 204-205.

Horie K, Kokubu C, Yoshida J, Akagi K, Isotani A, Oshitani A, Yusa K, Ikeda R, Huang Y, Bradley A, Takeda J. (2011) A homozygous mutant embryonic stem cell bank applicable for phenotype- driven genetic screening. Nature Methods. 8(12): 10711077.

Jennison M.J. (1937) The Relations Between Plate Counts and Direct Microscopic Counts of Escherichia coli During the Logarithmic Growth Period. J Bacteriol 33(5): 461-477. 
Lorenz A, Tuozzolo C, Louge MY. (1997) Measurements of impact properties of small, nearly spherical particles. Experimental Mechanics 37, 292-298.

Mills K, Gareau J, Garcia A. (2005) Low-cost modification to the Copaca- bana method for spreading transformation mixtures. Biotechniques 39(2): 188-188.

Nagler J, Krieger M, Linke M, Schonke J, Wiersig J. (2007) Leaking billiard. Phys Rev E 75046204.

Ragheb MN, Thomason MK, Hsu C, Nugent P, Gage J, Samadpour AN, Kariisa A, Merrikh CN, Miller SI, Sherman DR, Merrikh H. (2019) Inhibiting the Evolution of Antibiotic Resistance. Molecular Cell 73, 157-165.

Sanders ER (2012). Aseptic laboratory techniques: plating methods. Journal of visualized experiments. JoVE 63:e3064 1-7.

Strachan T, Read A. (2011) Amplifying DNA: Cell-based DNA Cloning and PCR. In: Human Molecular Genetics 4th Edition. Garland Science, Taylor \& Francis; 163-190.

Tomasiewicz D.M., Hotchkiss D.K., Reinbold G.W., Read R.B., Hartman P.A. (1980) The suitable number of colonies on plates for counting J. Food Prot., 43: 282-286.

Whitehead E., Rudolf F., Kaltenbach H.M., Stelling J. (2018) Automated Planning Enables Complex Protocols on Liquid-Handling Robots. ACS Synth Biol. 16; 7(3): 922932.

Wognum B, Yuan N, Lai B, Miller CL. (2013) Colony forming cell assays for human hematopoietic progenitor cells. In: Methods in molecular biology. vol. 946. Springer; p. 267-283.

Worthington M, Luo R, Pelo J. (2001) Copacabana method for spreading E-coli and yeast colonies. Biotechniques 30(4): 738-742.

\section{Supplementary material}

\section{Plating yeast Saccharomyces cerevisiae}

The yeast Saccharomyces cerevisiae colonies were grown at $30^{\circ} \mathrm{C}$ on rich media (Yeast Peptone Dextrose agar) [1]. The yeast culture was diluted with liquid YPD and 6 serial dilutions (in 1:10 dilution steps) were prepared in sterile media. The last three dilutions with concentrations of $10^{2} \mathrm{ppm}, 10^{1} \mathrm{ppm}$, and $10^{0} \mathrm{ppm}$, were used for cell 
plating. Concentration of $1 \mathrm{ppm}$ corresponds to $1 \mathrm{I}$ of yeast cultures per $1 \mathrm{I}$ of water. $100 \mu l$ of the diluted cell suspension was pipetted on to solid media in plates, then 10 glass beads were added and the plates were agitated. The plates were moved on a trajectory resembling the horizontally reflected letter "L": up- right-left-down. The number of plate movements was varied: either 5 "L" loops or 10 "L" loops. Plates containing the CFUs were incubated for $72 \mathrm{~h}$ at $30 \mathrm{C}$.

Supplementary Figure 1 (a) shows colony growth after $72 \mathrm{~h}$ incubation for selected plates. For a higher concentration we observed a good distribution of colonies in all replicas. For the concentration of $10^{\circ} \mathrm{pmm}$, one of the plates had no viable colonies for both configurations. Supplementary Figure 1 (b) shows the number of isolated colonies as a function of concentration and plating configuration.

[1] Dymond JS. Saccharomyces Cerevisiae Growth Media. In: Lorsch J, editor. Laboratory Methods in Enzymology: Cell, Lipid and Carbohydrate. vol. 533 of Methods in Enzymology. Elsevier Academic Press Inc; 2013. p. 191-204. 
Figure 1. Flowchart representing image processing. First, image was analysed to detect plate boundaries. Second, positions of CFUs were detected by fitting centroids.

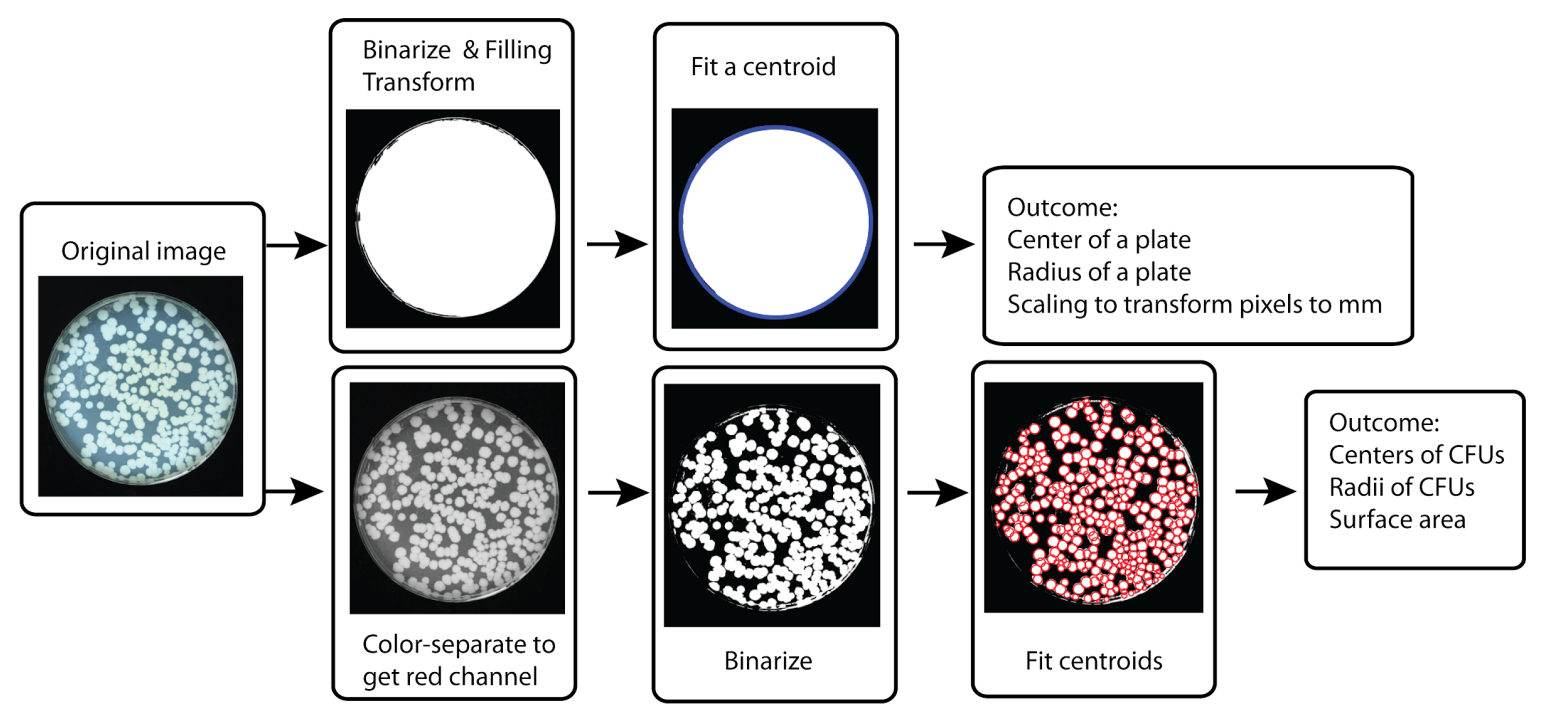


Figure 2. Metrics to assess the effectiveness of cell plating: $(A)$ Counting the number of colonies, $n_{C F U s}$. (B) Calculating the mean (solid line) and median (dashed line) of the distribution of distances to nearest colony. (C) Dividing plate into $5^{\circ}$ arcs, determining the number of colonies in that arc, and calculating coefficient of variation, $C V_{S}$. Black line shows expected distribution. (D) Calculating the mean difference between observed and expected distances from colonies and plate center, $m_{S}$; black line shows frequency based on a triangular distribution.

(A)

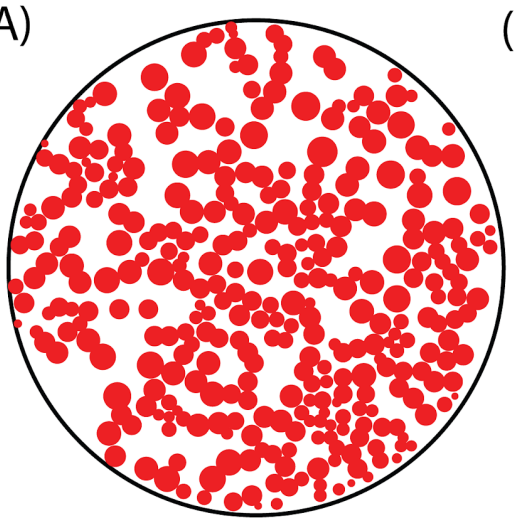

(C)

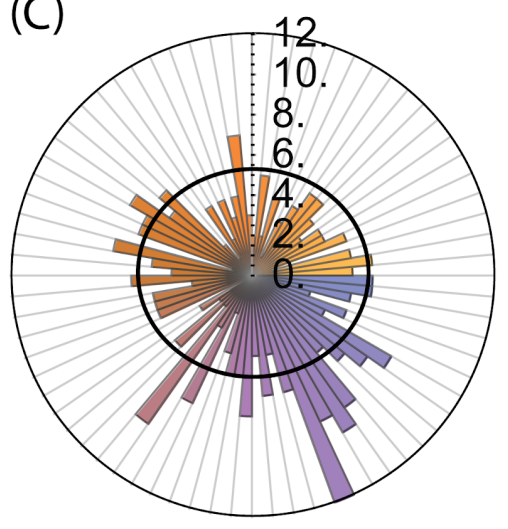

(B)

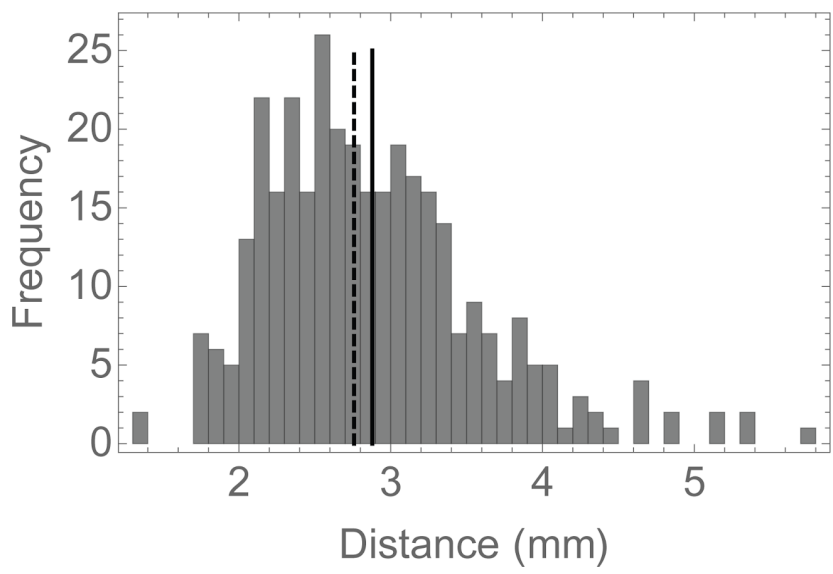

(D)

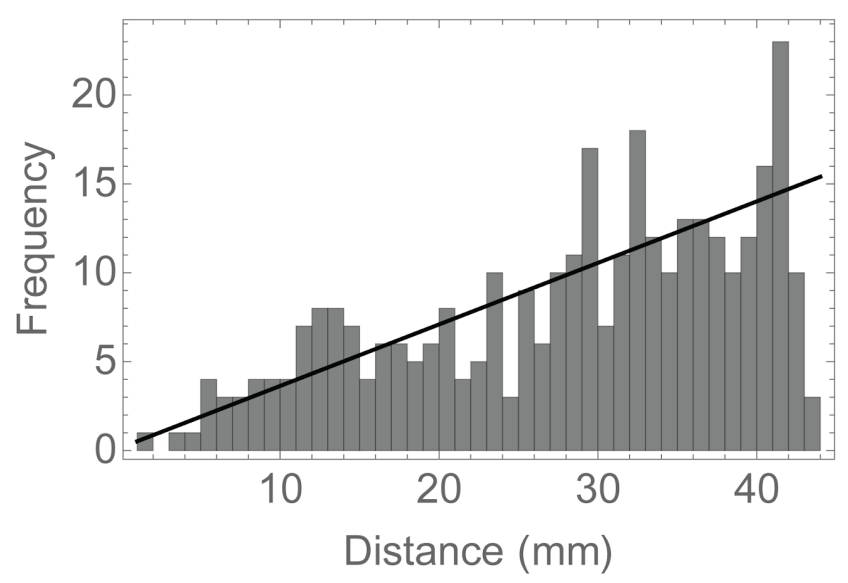


Figure 3. Experimental images of E.coli colonies and density histograms of simulated particle trajectories for the up-down movement. Columns correspond to a different number of glass beads. Blue circles indicate the initial positions of simulated particles.

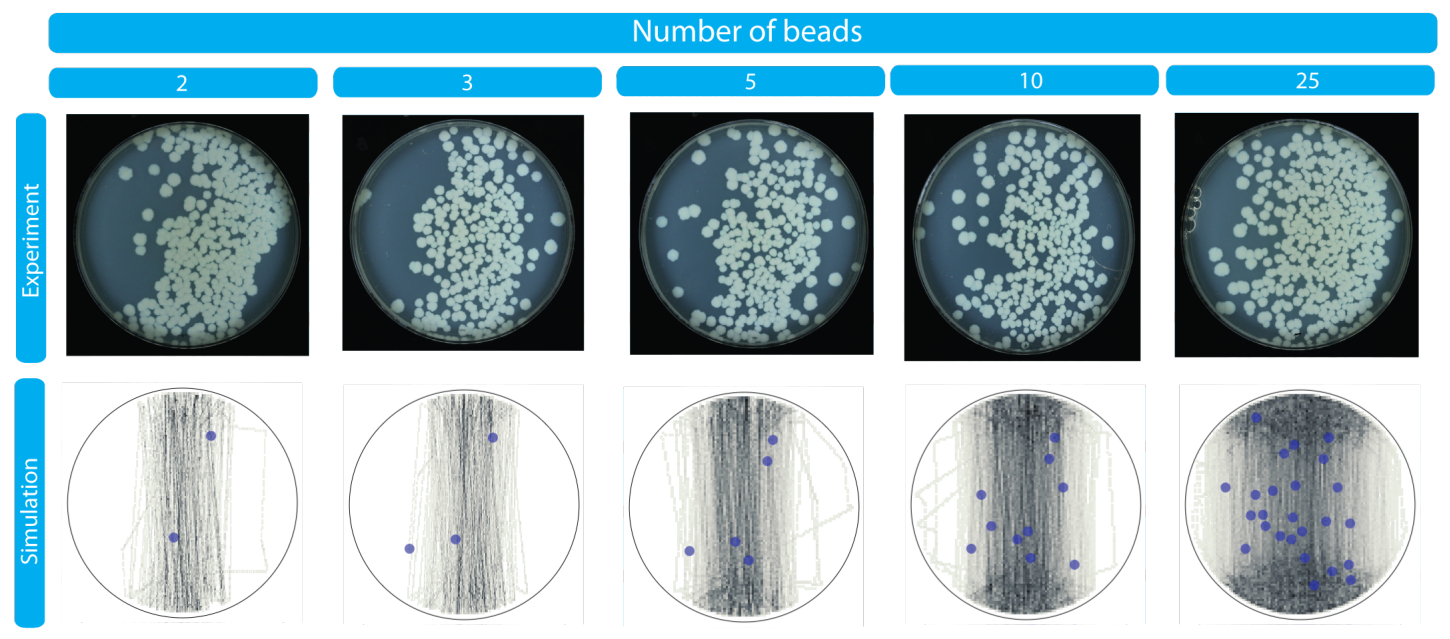


Figure 4. Experimental images of E.coli colonies for the L-shape movement. Columns correspond to a different number of glass beads, and rows correspond to a different number of loops.

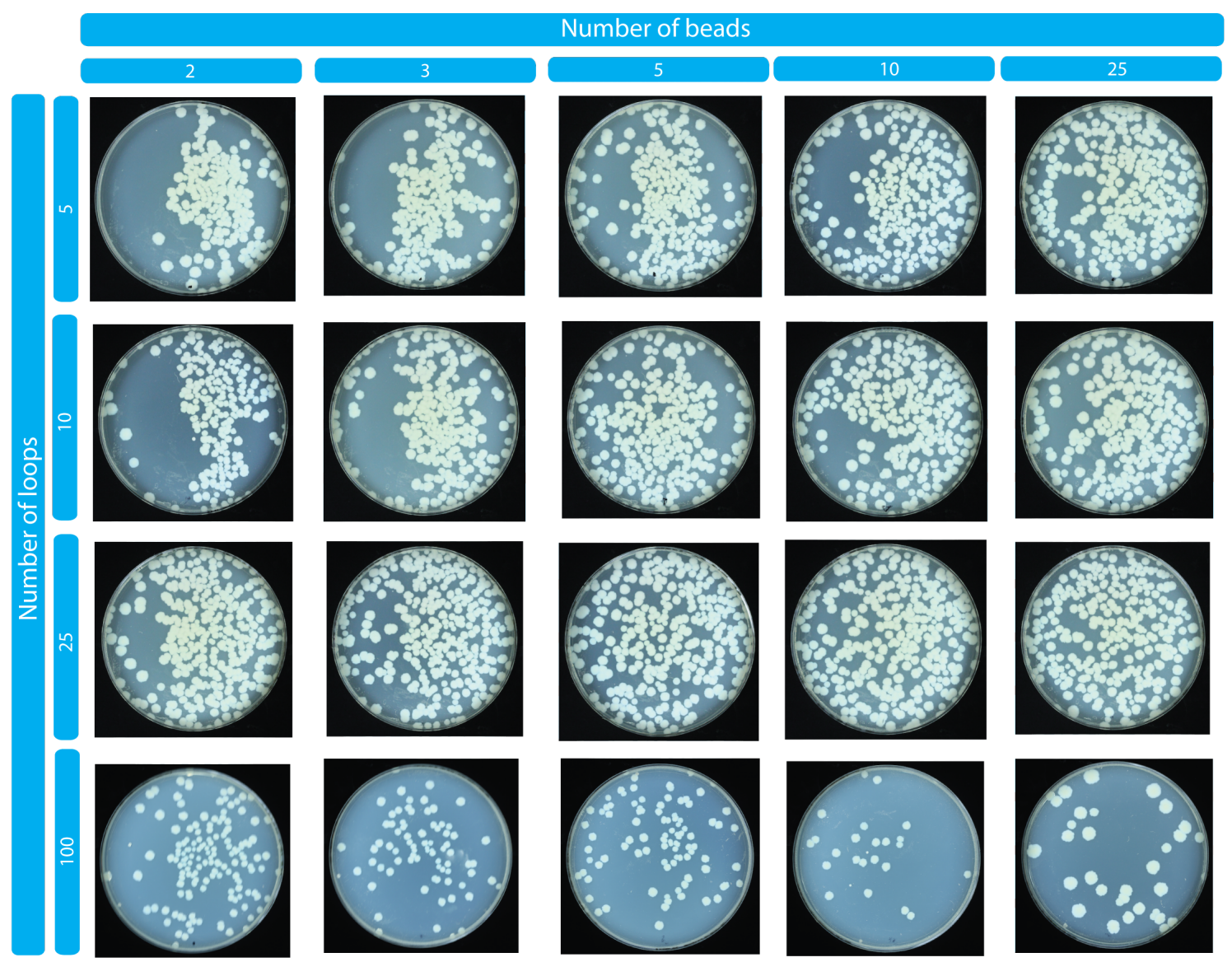


Figure 5. Density histograms of simulated particle trajectories for the L-shape movement. Columns correspond to a different number of glass beads, and rows correspond to a different number of loops. Blue circles indicate the initial positions of particles.

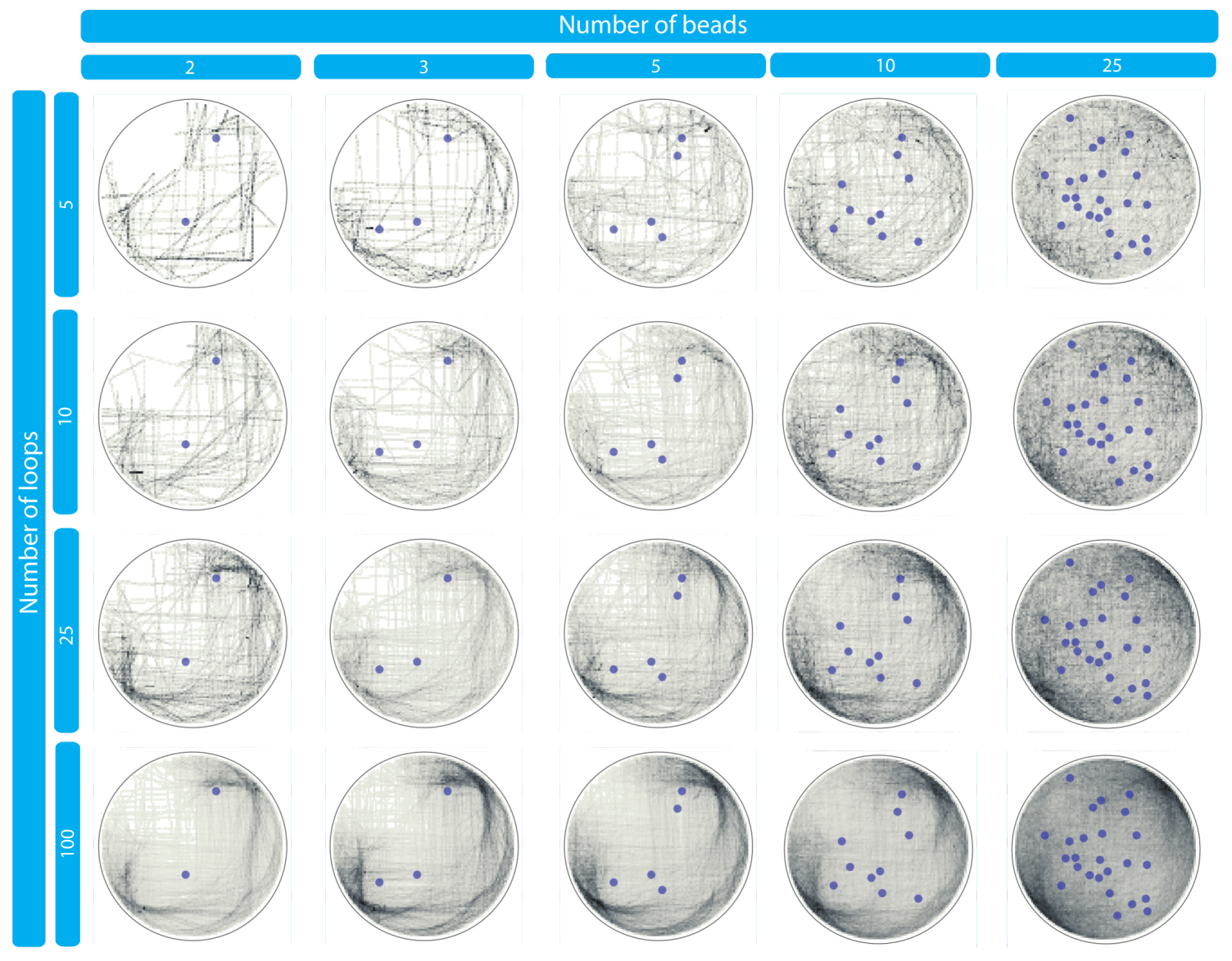



was not certified by peer review) is the author/funder. All rights reserved. No reuse allowed without permission.

Table 1. Results of the dilution experiment. The results show values of number of colonies, $n_{C F U}$; normalised mean distance to nearest colony, $d_{N B}$; variability and uniformity in colony spatial distribution on a plate, $C V_{S}$ and $m_{S}$ respectively.

\begin{tabular}{|c|c|c|c|c|}
\hline Dilution & $\mathbf{n}_{\text {CFUs }}$ & $\mathbf{d}_{\mathrm{NB}}$ & $\mathrm{CV}_{\mathrm{s}}$ & $\mathrm{m}_{\mathrm{s}}$ \\
\hline \multicolumn{5}{|l|}{5 loops } \\
\hline $10^{-5}$ & 946 & 2.71 & 0.590 & 7.760 \\
\hline $10^{-6}$ & 286 & 0.65 & 0.750 & 3.390 \\
\hline $10^{-7}$ & 59 & 0.1 & 1.200 & 1.090 \\
\hline \multicolumn{5}{|l|}{10 loops } \\
\hline $10^{-5}$ & 1050 & 3.23 & 0.450 & 8.770 \\
\hline $10^{-6}$ & 382 & 0.8 & 0.620 & 3.590 \\
\hline $10^{-7}$ & 71 & 0.13 & 0.970 & 1.340 \\
\hline
\end{tabular}


Table 2. Experimental results. Motions analysed are: L-shape (L), hourglass (HG), up-down (UD). The results show values of number of colonies, $n_{C F U}$; normalised mean distance to nearest colony, $d_{N B}$; variability and uniformity in colony spatial distribution on a plate, $C V_{S}$ and $m_{S}$ respectively. In each column darker shade corresponds to higher value.

\begin{tabular}{|c|c|c|c|c|c|c|}
\hline \multicolumn{3}{|c|}{ Parameters } & \multicolumn{4}{|c|}{ Observations } \\
\hline Motion & $\begin{array}{c}\text { No. } \\
\text { Beads }\end{array}$ & $\begin{array}{l}\text { No. } \\
\text { Loops }\end{array}$ & $\mathbf{n}_{\text {CFUs }}$ & $C V_{s}$ & $\mathbf{m}_{\mathbf{s}}$ & $\mathbf{d}_{\mathrm{NB}}$ \\
\hline$\overline{\mathbf{L}}$ & 2 & 5 & 230 & 0.90 & 3.07 & 0.5 \\
\hline $\mathbf{L}$ & 2 & 10 & 254 & 0.77 & 3.05 & 0.51 \\
\hline $\mathbf{L}$ & 2 & 25 & 321 & 0.62 & 2.16 & 0.58 \\
\hline $\mathbf{L}$ & 2 & 100 & 162 & 0.66 & 1.72 & 0.54 \\
\hline $\mathbf{L}$ & 3 & 5 & 230 & 0.89 & 3.00 & 0.49 \\
\hline $\mathbf{L}$ & 3 & 10 & 236 & 0.60 & 2.80 & 0.53 \\
\hline $\mathbf{L}$ & 3 & 25 & 338 & 0.61 & 1.98 & 0.57 \\
\hline $\mathbf{L}$ & 3 & 100 & 121 & 0.78 & 2.01 & 0.48 \\
\hline $\mathbf{L}$ & 5 & 5 & 273 & 0.77 & 3.18 & 0.53 \\
\hline $\mathbf{L}$ & 5 & 10 & 315 & 0.60 & 2.88 & 0.56 \\
\hline $\mathbf{L}$ & 5 & 25 & 333 & 0.42 & 2.44 & 0.61 \\
\hline $\mathbf{L}$ & 5 & 100 & 99 & 0.79 & 1.54 & 0.73 \\
\hline $\mathbf{L}$ & 10 & 5 & 316 & 0.54 & 2.68 & 0.56 \\
\hline $\mathbf{L}$ & 10 & 10 & 287 & 0.57 & 1.85 & 0.58 \\
\hline $\mathbf{L}$ & 10 & 25 & 325 & 0.44 & 2.17 & 0.61 \\
\hline $\mathbf{L}$ & 10 & 100 & 38 & 1.25 & 0.77 & 0.45 \\
\hline $\mathbf{L}$ & 25 & 5 & 327 & 0.44 & 2.72 & 0.6 \\
\hline $\mathbf{L}$ & 25 & 10 & 271 & 0.54 & 1.94 & 0.58 \\
\hline $\mathbf{L}$ & 25 & 25 & 299 & 0.48 & 2.47 & 0.59 \\
\hline $\mathbf{L}$ & 25 & 100 & 20 & 1.84 & 0.55 & 0.93 \\
\hline HG & 2 & 5 & 232 & 0.81 & 3.37 & 0.5 \\
\hline HG & 2 & 10 & 230 & 0.60 & 3.04 & 0.55 \\
\hline HG & 2 & 25 & 253 & 0.56 & 3.18 & 0.57 \\
\hline HG & 3 & 5 & 186 & 0.94 & 2.69 & 0.99 \\
\hline HG & 3 & 10 & 289 & 0.50 & 3.81 & 0.57 \\
\hline HG & 3 & 25 & 276 & 0.50 & 2.44 & 0.61 \\
\hline HG & 5 & 5 & 230 & 0.83 & 2.12 & 0.52 \\
\hline HG & 5 & 10 & 289 & 0.66 & 2.91 & 0.55 \\
\hline HG & 5 & 25 & 248 & 0.52 & 1.93 & 1.2 \\
\hline HG & 10 & 5 & 229 & 0.68 & 2.60 & 0.57 \\
\hline HG & 10 & 10 & 281 & 0.57 & 2.90 & 0.57 \\
\hline HG & 10 & 25 & 232 & 0.51 & 2.04 & 0.59 \\
\hline HG & 25 & 5 & 236 & 0.68 & 2.25 & 0.52 \\
\hline HG & 25 & 10 & 299 & 0.50 & 2.78 & 0.57 \\
\hline HG & 25 & 25 & 74 & 0.99 & 1.08 & 0.47 \\
\hline UD & 2 & 50 & 387 & 0.79 & 2.70 & 0.52 \\
\hline UD & 3 & 50 & 413 & 0.67 & 5.34 & 0.51 \\
\hline UD & 5 & 50 & 396 & 0.67 & 3.94 & 0.54 \\
\hline UD & 10 & 50 & 416 & 0.67 & 4.66 & 0.53 \\
\hline UD & 25 & 50 & 430 & 0.78 & 3.74 & 0.5 \\
\hline
\end{tabular}


Supplementary Figure 1. Plating results for S.cerevisiae. (a) Grown colonies after $72 \mathrm{~h}$ incubation period. (b) The number of colonies as a function of the concentration and the plating method: 5 loops (black circles) and 10 loops (gray squares).

(a)

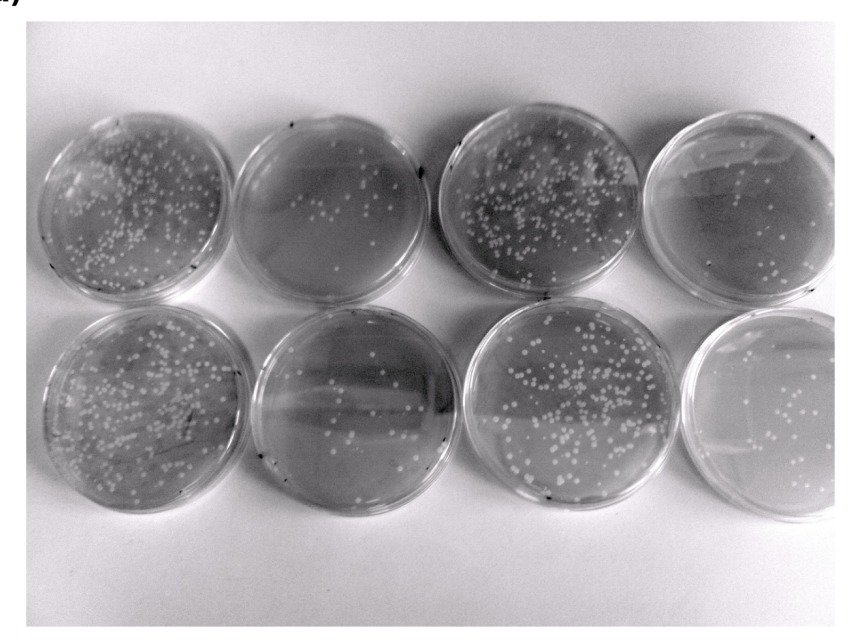

(b)

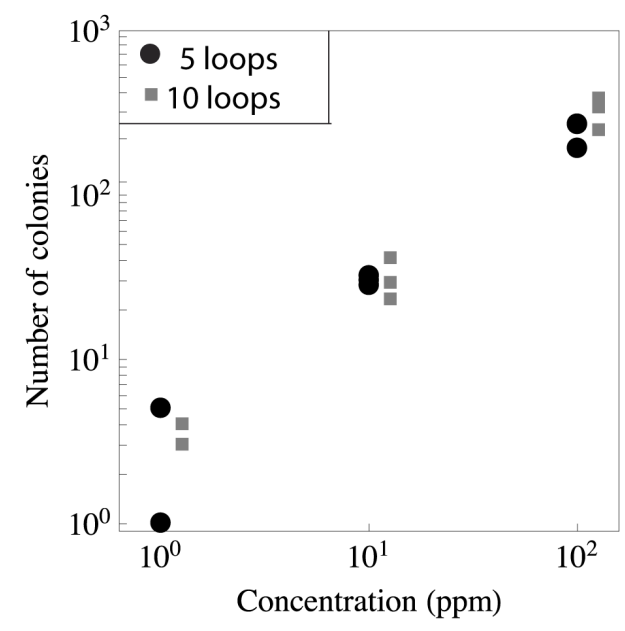

